\title{
ADAPTIVE CONTROL FOR SEMILINEAR STOCHASTIC SYSTEMS*
}

\author{
T. E. DUNCAN ${ }^{\dagger}$, B. MASLOWSKI ${ }^{\ddagger}$, AND B. PASIK-DUNCAN $^{\dagger}$
}

\begin{abstract}
An adaptive, ergodic cost stochastic control problem for a partially known, semilinear, stochastic system in an infinite dimensional space is formulated and solved. The solutions of the Hamilton-Jacobi-Bellman equations for the discounted cost and the ergodic cost stochastic control problems require some special interpretations because they do not typically exist in the usual sense. The solutions of the parameter dependent ergodic Hamilton-Jacobi-Bellman equations are obtained from some corresponding discounted cost control problems as the discount rate tends to zero. The solutions of the ergodic Hamilton-Jacobi-Bellman equations are shown to depend continuously on the parameter. A certainty equivalence adaptive control is given that is based on the optimal controls from the solutions of the ergodic Hamilton-Jacobi-Bellman equations and a strongly consistent family of estimates of the unknown parameter. This adaptive control is shown to achieve the optimal ergodic cost for the known system.
\end{abstract}

Key words. stochastic adaptive control, ergodic control, stochastic semilinear systems, stochastic optimal control, distributed parameter systems

AMS subject classifications. 93C40, 93C20, 60J27, 60H15

\section{PII. S0363012999351826}

1. Introduction. Ergodic cost stochastic control problems for finite dimensional, nonlinear stochastic systems have been investigated for more than two decades (e.g., [2], [9], [25], and [1], [28], and the references therein). There has been some work on the corresponding adaptive control problem for these partially known stochastic systems. In [3], [4], [5] some properties of the solution of the Hamilton-JacobiBellman (HJB) equation of optimal control are used to verify self-optimality of an adaptive control using a strongly consistent family of estimates of the unknown parameters. In [17] an almost optimal adaptive control is constructed for a partially known nonlinear stochastic system. To obtain an optimal feedback control in an explicit form, the associated HJB equation must be solved, and for an adaptive control problem, a continuous dependence of the solution of the HJB equation must be verified. Some results for an infinite time horizon discounted cost control problem for a semilinear stochastic system in an infinite dimensional state space are given in [22], where the HJB equation is considered in the mild form, and in [23], where a viscosity solution of the HJB equation is used. Some results for an ergodic cost control problem for a semilinear stochastic system in an infinite dimensional state space are given in [20], where the HJB equation is solved. This is apparently the only result for ergodic cost control for semilinear stochastic systems using the HJB equation. Some other approaches and results for ergodic cost control are given in [16], [18]. For adaptive control, there are results for linear stochastic systems with an ergodic quadratic cost in [13], [14], [15]. Since the results for the discounted cost and the ergodic cost stochastic control problems for known semilinear stochastic systems are relatively recent, it appears that this is the first work on adaptive control for stochastic semilinear systems.

\footnotetext{
*Received by the editors February 11, 1999; accepted for publication (in revised form) November 29, 1999; published electronically June 15, 2000. This research was supported in part by NSF grant DMS 9623439 and GACR grant 201/98/1454.

http://www.siam.org/journals/sicon/38-6/35182.html

${ }^{\dagger}$ Department of Mathematics, University of Kansas, Lawrence, KS 66045 (duncan@math.ukans. edu, bozenna@math.ukans.edu).

${ }^{\ddagger}$ Institute of Mathematics, Academy of Sciences, Prague, Czech Republic (maslow@math.cas.cz).
} 
In this paper an adaptive, ergodic cost control problem is solved for a semilinear stochastic system in an infinite dimensional state space using a solution of the HJB equation. An adaptive control is obtained from the certainty equivalence principle and the optimal control from the solution of the HJB equation. Continuity of the optimal cost with respect to the parameter is shown and the adaptive control is verified to be self-optimal.

A brief outline of the paper is given now. In section 2, the adaptive control problem is formulated and the assumptions are described. An example of a controlled stochastic parabolic partial differential equation is given for which the assumptions are satisfied. Some preparatory results for the analysis of the adaptive control problem are given. For example, the tightness of the probability laws of the solutions of the controlled semilinear systems and a uniform boundedness of their moments are verified. Some uniform bounds on the derivatives of the Markov transition semigroup for the uncontrolled system are also verified. In section 3, the parameter estimation problem is considered. In this section it is assumed that the unknown parameter appears affinely in the stochastic system. With an identifiability condition, it is shown that a family of least squares estimates is strongly consistent. In section 4, the self-optimality of an adaptive control is verified. Initially the parameter dependent, infinite dimensional HJB equations for the ergodic and discounted costs control problems are formally introduced. Their solutions are defined by the generator of an Ornstein-Uhlenbeck semigroup. A family of suitably normalized solutions of the HJB equations for the discounted costs are shown to be relatively compact for arbitrarily small discount rates and all values of the parameter in the Sobolev space $W^{1,2}(H, \mu)$, where $H$ is the Hilbert space that is the state space for the system and $\mu$ is a limiting Gaussian measure for the solution of the associated uncontrolled linear system (Ornstein-Uhlenbeck process). This relative compactness property provides the ergodic control result as a suitable limit of the discounted control results as the discount rate tends to zero. The proof of the relative compactness uses a method in [20] for known systems in a space of continuous, polynomially bounded functions where an upper bound on the norm of the controls is required. The existence and the uniqueness of the solution of the ergodic HJB equation and its relation to an optimal control and the optimal cost are given. While the existence of the solution follows from a result in [20], the uniqueness of the solution in $W^{1,2}(H, \mu)$ is new. The continuous dependence of the solutions of the ergodic HJB equations on the parameter in the $W^{1,2}(H, \mu)$ is verified. A certainty equivalence adaptive control is defined that is based on the optimal controls from the solutions of the ergodic HJB equations and a strongly consistent family of estimates of the unknown parameter. The main results of section 4, Theorem 4.1 and Corollary 4.2, state the self-optimality of this adaptive control, that is, this adaptive control achieves the optimal ergodic cost for the true system.

2. Preliminaries. Let $(X(t), t \geq 0)$ be an $H$-valued, parameter dependent, controlled process that satisfies the stochastic differential equation

$$
\begin{aligned}
d X(t) & =(A X(t)+f(\alpha, X(t))-u(t)) d t+Q^{1 / 2} d W(t) \\
X(0) & =x
\end{aligned}
$$

where $H$ is a real, separable Hilbert space with inner product $\langle\cdot, \cdot\rangle$ and norm $|\cdot|$, $A: \operatorname{Dom}(A) \rightarrow H$ is a densely defined, unbounded linear operator on $H, f(\alpha, \cdot)$ : $H \rightarrow H$ for each $\alpha \in \mathcal{A} \subset \mathbb{R}^{q}$ that is a compact set of parameters, $(W(t), t \geq 0)$ is a 
standard, cylindrical $H$-valued Wiener process defined on a filtered probability space $\left(\Omega, \mathcal{F},\left(\mathcal{F}_{t}\right), \mathbb{P}\right)$ and $Q^{1 / 2} \in \mathcal{L}(H)$. The family of admissible controls is

$$
\mathcal{U}=\left\{u: \mathbb{R}_{+} \times \Omega \rightarrow B_{R} \mid u \text { is measurable and }\left(\mathcal{F}_{t}\right) \text { adapted }\right\},
$$

where $B_{R}=\{y \in H|| y \mid \leq R\}$ and $R>0$ is fixed. A family of Markov controls, e.g., $u(t)=\tilde{u}(X(t))$, is also considered where $\tilde{u} \in \tilde{\mathcal{U}}$ and

$$
\tilde{\mathcal{U}}=\left\{\tilde{u}: H \rightarrow B_{R} \mid \tilde{u} \text { is Borel measurable }\right\} .
$$

The cost functionals $J(x, \lambda, u)$ and $\tilde{J}(x, u)$ are given as

$$
J(x, \lambda, u)=\mathbb{E}_{x, u} \int_{0}^{\infty} e^{-\lambda t}(\psi(X(t))+h(u(t))) d t
$$

and

$$
\tilde{J}(x, u)=\liminf _{T \rightarrow \infty} \mathbb{E}_{x, u} \frac{1}{T} \int_{0}^{T}(\psi(X(t))+h(u(t))) d t,
$$

where $\lambda>0, h: B_{R} \rightarrow \mathbb{R}_{+}$, and $\psi: H \rightarrow \mathbb{R}$, that describe a discounted and an ergodic control problem, respectively.

The adaptive control problem is to find a family of strongly consistent estimates of the unknown parameter $\alpha$ and to determine an adaptive control from the family of admissible controls such that the optimal ergodic cost, $\inf _{u \in \mathcal{U}} \tilde{J}(x, u)$, is achieved.

The following assumptions are selectively used in the paper.

(A1) The linear operator $Q=Q^{1 / 2^{*}} Q^{1 / 2}$ is invertible, $Q^{-1} \in \mathcal{L}(H)$ and $(S(t), t \geq$ 0 ), where $S(t)=e^{t A}$, is an exponentially stable semigroup of contractions, that is,

$$
\|S(t)\|_{\mathcal{L}(H)} \leq e^{-\omega t}
$$

for all $t \geq 0$ and some $\omega>0$. Furthermore, the semigroup is Hilbert-Schmidt and there is a $\gamma>0$ such that

$$
\int_{0}^{T} t^{-\gamma}\|S(t)\|_{\mathrm{HS}}^{2} d t<\infty
$$

for some $T>0$, where $\|\cdot\|_{\text {HS }}$ is the Hilbert-Schmidt norm.

(A2) The function $f(\alpha, \cdot): H \rightarrow H$ is Lipschitz continuous and Gâteaux differentiable. The Gâteaux derivative $D f(\alpha, \cdot) h$ is continuous on $H$ for each $h \in H$ and $\alpha \in \mathcal{A}$ and there is a $\beta \in \mathbb{R}$ such that

$$
\langle D f(\alpha, x) h, h\rangle \leq \beta|h|^{2}
$$

for all $x \in H, h \in H$, and $\alpha \in \mathcal{A}$.

(A3) The function $f(\cdot, x): \mathcal{A} \rightarrow H$ is continuous for each $x \in H$ and there are constants $p>0$ and $C>0$ such that

$$
|f(\alpha, x)| \leq C\left(1+|x|^{p}\right)
$$

for each $x \in H$ and $\alpha \in \mathcal{A}$.

(A4) $\psi \in C_{b}(H)$. 
(A5) The function $h: H \rightarrow \mathbb{R}$ is convex and bounded on bounded sets and continuous. The function $\tilde{H}: H \rightarrow \mathbb{R}$ given by $\tilde{H}(x)=\sup _{|y| \leq R}[\langle y, x\rangle-h(y)]$ is continuously Fréchet differentiable.

Some implications of the assumptions (A1)-(A5) are described now. Consider the linear stochastic differential equation obtained from (2.1) by choosing $f \equiv 0$ and $u \equiv 0$, that is,

$$
\begin{aligned}
d Z(t) & =A Z(t) d t+Q^{1 / 2} d W(t), \\
Z(0) & =x .
\end{aligned}
$$

It is well known (e.g., [11]) that if (A1) is satisfied then (2.6) has a unique mild solution

$$
Z(t)=S(t) x+\int_{0}^{t} S(t-r) Q^{1 / 2} d W(r)
$$

which is an $H$-valued process with continuous sample paths, is ergodic, and has a unique invariant probability measure

$$
\mu=N\left(0, Q_{\infty}\right)
$$

where

$$
Q_{\infty}=\int_{0}^{\infty} S(r) Q S^{*}(r) d r
$$

is a trace class operator on $H$. If (A2) is satisfied, then (2.1) has a unique mild solution

$$
X(t)=S(t) x+\int_{0}^{t} S(t-r)(f(\alpha, X(r))-u(r)) d r+\int_{0}^{t} S(t-r) Q^{1 / 2} d W(r)
$$

for each $u \in \mathcal{U}$ and $\alpha \in \mathcal{A}$. If the control in (2.1) has the feedback form $u(t)=\tilde{u}(X(t))$, where $\tilde{u} \in \tilde{\mathcal{U}}$, then the solution of (2.1) is obtained by an absolute continuity of measures as a weak solution in the probabilistic sense. More specifically, if $\mathbb{P}$ is the probability measure for the solution of $(2.1)$ with $u \equiv 0$, then the probability measure $\mathbb{P}_{\tilde{u}}$ for the solution of

$$
\begin{aligned}
d X(t) & =(A X(t)+f(\alpha, X(t))-\tilde{u}(X(t))) d t+Q^{1 / 2} d W(t), \\
X(0) & =x
\end{aligned}
$$

is induced from $\mathbb{P}$ by the Radon-Nikodým derivative

$$
\frac{d \mathbb{P}_{\tilde{u}}}{d \mathbb{P}}=\exp \left[-\int_{0}^{T}\left\langle Q^{-1 / 2} \tilde{u}(X(s)), d W(s)\right\rangle-\frac{1}{2} \int_{0}^{T}\left|Q^{-1 / 2} \tilde{u}(X(s))\right|^{2} d s\right] .
$$

The assumption (A3) is used to verify a suitable continuous dependence of the solutions of the ergodic HJB equations on the parameter which is important to verify the self-optimality of a certainty equivalence adaptive control. The assumptions (A4) and (A5) are standard conditions on a cost functional in the stochastic control of semilinear systems (e.g., [20], [22]). Note that (A5) is satisfied in the case where $h(x)=|x|^{2}$ so that $\tilde{H}(x)=\hat{H}(|x|)$, where

$$
\hat{H}(r)= \begin{cases}\frac{r^{2}}{4} & \text { if }|r| \leq 2 R \\ R|r|-R^{2} & \text { if }|r|>2 R\end{cases}
$$


To provide some additional perspective for the adaptive control problem, an example of a stochastic partial differential equation that satisfies the assumptions is given. Consider the stochastic partial differential equation

$$
\frac{\partial y}{\partial t}(t, \xi)=\frac{\partial^{2} y}{\partial \xi^{2}}(t, \xi)+F(\alpha, y(t, \xi))-u(t, \xi)+\eta(t, \xi)
$$

for $(t, \xi) \in \mathbb{R}_{+} \times(0,1)$ with the initial condition $y(0, \xi)=y_{0}(\xi), \xi \in(0,1)$ and the Dirichlet boundary conditions

$$
y(t, 0)=y(t, 1)=0
$$

for $t \geq 0$. The function $F: \mathcal{A} \times \mathbb{R} \rightarrow \mathbb{R}$ satisfies the following: $F(\cdot, y)$ is continuous for each $y \in \mathbb{R}, F(\alpha, \cdot)$ is (globally) Lipschitz continuous for each $\alpha \in \mathcal{A},|F(\alpha, y)| \leq$ $C(1+|y|)$ for some $C>0$ and all $\alpha \in \mathcal{A}$ and $y \in \mathbb{R}$, and $F^{\prime}(\alpha, y) \leq \beta$ for some $\beta \in \mathbb{R}$, and the term $\eta$ formally denotes a space time white noise. The control $(u(t), t \geq 0)$ is assumed to be adapted to the noise process and to take values in a ball, $B_{R}$, in $L^{2}(0,1)$. The formal equation $(2.12)$ can be rigorously described in a standard way as an equation of the form (2.1) in the Hilbert space $H=L^{2}(0,1)$, $A=\partial^{2} / \partial \xi^{2}, \operatorname{Dom}(A)=\left\{\varphi \in L^{2}(0,1) \mid \varphi, \varphi^{\prime}\right.$ are absolutely continuous, $\varphi^{\prime \prime} \in L^{2}(0,1)$, $\varphi(0)=\varphi(1)=0\}, f(\alpha, x)(\xi)=F(\alpha, x(\xi))$ for $x \in H, \alpha \in \mathcal{A}, \xi \in(0,1)$ and a cylindrical Wiener process with $Q=\delta I$ where $\delta>0$ is a constant and $I$ is the identity on $I$. For $\psi$ and $h$ in the cost functionals $(2.4),(2.5)$ arbitrary $\psi \in C_{b}\left(L^{2}(0,1)\right)$ and $h: L^{2}(0,1) \rightarrow \mathbb{R}_{+}$satisfying (A5) can be chosen, e.g., $h(u)=|u|^{2}$. It is well known that all of the assumptions (A1)-(A5) are satisfied where $\gamma \in(0,1 / 4)$ in (A1).

It is convenient to denote by $\mathbb{P}_{x, u}^{\alpha}$ the probability measure on $\Omega$ for the solution of (2.1) with $X(0)=x$, a control $u \in \mathcal{U}$, and a parameter $\alpha \in \mathcal{A}$. Let $\mathbb{E}_{x, u}^{\alpha}$ be the expectation for the probability $\mathbb{P}_{x, u}^{\alpha}$.

In the following proposition some stability, boundedness, and tightness properties are given.

Proposition 2.1. If (A1) and (A2) are satisfied, where $\omega-\beta>0$, then the following apply.

(i) For each $p^{\prime}>0$ and $T>0$ the following inequalities are satisfied:

$$
\sup _{\alpha \in \mathcal{A} u \in \mathcal{U}} \mathbb{E}_{x, u}^{\alpha}\left[\sup _{0 \leq t \leq T}|X(t)|^{2 p^{\prime}}\right] \leq C_{T}|x|^{2 p^{\prime}}+\tilde{C}_{T}
$$

and

$$
\sup _{t \in \mathbb{R}_{+}} \sup _{\alpha \in \mathcal{A}} \sup _{u \in \mathcal{U}} \mathbb{E}_{x, u}^{\alpha}\left[|X(t)|^{2 p^{\prime}}\right] \leq C_{1}|x|^{2 p^{\prime}}+C_{2} .
$$

(ii) For each $x \in H, \alpha \in \mathcal{A}$, and $u \in \mathcal{U}$ the following inequality is satisfied:

$$
\limsup _{t \rightarrow \infty} \frac{1}{t} \int_{0}^{t}|X(s)|^{2} d s<\infty \quad \text { almost surely (a.s.) } \mathbb{P}_{x, u}^{\alpha} \text {. }
$$

(iii) For each $x \in H, \alpha \in \mathcal{A}$, and $u \in \mathcal{U}$ there is a sequence $\left(K_{n}, n \in \mathbb{N}\right)$ of compact sets in $H$ such that

$$
\lim _{n \rightarrow \infty} \sup _{t \geq 1} \mathbb{P}_{x, u}^{\alpha}\left(X(t) \in H \backslash K_{n}\right)=0,
$$

so the family of measures $\left(\mu_{X(t)}, t \geq 1\right)$ is tight where $\mu_{X(t)}$ is the probability law for $X(t)$. 
Proof. To verify (i) the solution of (2.1) can be expressed as

$$
X(t)=\mu(t, \tilde{Z})+\tilde{Z}(t),
$$

where

$$
\tilde{Z}(t)=-\int_{0}^{t} S(t-r) u(r) d r+\int_{0}^{t} S(t-r) Q^{1 / 2} d W(r)
$$

and $\mu(t, \varphi)$ is the solution of the deterministic integral equation

$$
\left.\mu(t, \varphi)=S(t) x+\int_{0}^{t} S(t-r) f(\alpha, \mu(r, \varphi))+\varphi(r)\right) d r
$$

where $\varphi \in C\left(\mathbb{R}_{+}, H\right)$ and $\varphi(0)=0$. Since the semigroup $(S(t), t \geq 0)$ is exponentially stable, it easily follows that

$$
\sup _{t \in \mathbb{R}_{+}} \mathbb{E}|\tilde{Z}(t)|^{m}<\infty
$$

for each $m>0$. Thus to verify (2.14), it suffices to show that

$$
\sup \mathbb{E}_{x, u}^{\alpha}\left[|\mu(t, \tilde{Z})|^{2 p^{\prime}}\right] \leq C\left(1+|x|^{2 p^{\prime}}\right)
$$

for some constant $C$ and the supremum is taken over all $t \in \mathbb{R}_{+}, u \in \mathcal{U}$, and $\alpha \in \mathcal{A}$. It can be assumed in (A2) that $\beta \leq 0$, for otherwise $\beta I$ can be subtracted from $f$ and added to $A$. Using the standard approximation of $f$ by the sequence $\left(f_{n}, n \in \mathbb{N}\right)$, where $f_{n}=(1 / n)\left[(I-n f)^{-1}-I\right]$, and using the Gronwall lemma it follows that

$$
|\mu(t, \varphi)|^{2 p^{\prime}} \leq e^{-2 p^{\prime} \omega t}|x|^{2 p^{\prime}}+2 p \int_{0}^{t} e^{-2 p^{\prime} \omega(t-s)}|f(\alpha, \varphi(s))||\mu(s, \varphi)|^{2 p^{\prime}-1} d s
$$

(cf. [20, Lemma 2.2] or [12] for a similar verification). By (A3) it follows that

$$
|f(\alpha, x)| \leq \tilde{C}\left(1+|x|^{p}\right)
$$

for all $x \in H$ and $\alpha \in \mathcal{A}$ and some constant $\tilde{C}$. If $p^{\prime}=1 / 2$, then (2.17) and (2.19) imply (2.18). Otherwise a verification by induction is made as follows. Assume that

$$
\sup _{t \in \mathbb{R}_{+}, \alpha \in \mathcal{A}, u \in \mathcal{U}} \mathbb{E}_{x, u}^{\alpha}\left[|\mu(t, \tilde{Z})|^{2 p^{\prime}-(1 / 2)}\right] \leq C_{3}|x|^{2 p^{\prime}-(1 / 2)}+C_{4}
$$

for all $x \in H$ and some constants $C_{3}$ and $C_{4}$. Using (2.19) and the Hölder inequality with exponents $q=\left[2 p^{\prime}-(1 / 2)\right] /\left(2 p^{\prime}-1\right)$ and $q^{\prime}=q /(q-1)$ it follows that

$$
\begin{aligned}
& \mathbb{E}_{x, u}^{\alpha}\left[|\mu(t, \tilde{Z})|^{2 p^{\prime}}\right] \leq e^{-2 p^{\prime} \omega t}|x|^{2 p^{\prime}}+C_{5} \int_{0}^{t} e^{-2 p^{\prime} \omega(t-s)}\left(\mathbb{E}_{x, u}^{\alpha}|f(\alpha, \tilde{Z}(s))|^{q^{\prime}}\right)^{1 / q^{\prime}} d s \\
& \leq C_{6}|x|^{2 p^{\prime}}+C_{7} \\
& \cdot\left(\mathbb{E}_{x, u}^{\alpha}|\mu(t, \tilde{Z})|^{2 p^{\prime}-(1 / 2)}\right)^{1 / q}
\end{aligned}
$$

for all $x \in H$ and some constants $C_{6}$ and $C_{7}$. This completes the verification of (2.14). It has been shown [30] that

$$
\mathbb{E}\left[\sup _{0 \leq t \leq T}|\tilde{Z}(t)|^{q}\right]<\infty
$$


for each $q>0$. Thus the inequality (2.13) follows from (2.19) in a similar way.

To verify (ii) the inequalities (2.19) and (2.20) imply that

$$
\frac{1}{t} \int_{0}^{t}|\mu(r, \varphi)|^{2} d r \leq \frac{C_{8}(x)}{t}+C_{9} \frac{1}{t} \int_{0}^{t}|\varphi(s)|^{2 p} d s
$$

for some $C_{8}(x)$ depending on $x \in H$ and constant $C_{9}$. Thus

$$
\begin{aligned}
\frac{1}{t} \int_{0}^{t}|X(s)|^{2} d s & \leq \frac{2}{t} \int_{0}^{t}|\mu(s, \tilde{Z})|^{2} d s+\frac{2}{t} \int_{0}^{t}|\tilde{Z}(s)|^{2} d s \\
& \leq \frac{2 C_{8}(x)}{t}+\frac{2 C_{9}}{t} \int_{0}^{t}|\tilde{Z}(s)|^{2 p} d s+\frac{2}{t} \int_{0}^{t}|\tilde{Z}(s)|^{2} d s
\end{aligned}
$$

Note that

$$
\frac{1}{t} \int_{0}^{t}\left|\int_{0}^{s} S(s-r) u(r) d r\right|^{2 q} d s \leq \frac{1}{t} \int_{o}^{t}\left(\frac{R}{\omega}\right)^{2 q} d s \leq\left(\frac{R}{\omega}\right)^{2 q}
$$

and

$$
\lim _{t \rightarrow \infty} \frac{1}{t} \int_{0}^{t}\left|\int_{0}^{s} S(s-r) Q^{1 / 2} d W(r)\right|^{2 q} d s=C(q)\left(\operatorname{Tr} Q_{\infty}\right)^{q} \quad \text { a.s. } \mathbb{P}_{x, u}^{\alpha}
$$

for any $q \in \mathbb{N}$, and $C(q)$ depends only on $q$. This equality follows because the Ornstein-Uhlenbeck process $\left(\int_{0}^{t} S(t-r) d W(r), t \geq 0\right)$ is ergodic and by the strong law of large numbers (cf. [27]) the family of time averages in (2.23) converge almost surely to the $2 q$ th moment of the invariant measure, $\mu=N\left(0, Q_{\infty}\right)$. The inequalities (2.21) and (2.22) and the equality (2.23) verify the inequality (2.15) in (ii).

To verify (iii) the solution $(X(t), t \geq 0)$ of $(2.1)$ is expressed as

$$
\begin{aligned}
X(t+1)= & S(1) X(t)+\int_{t}^{t+1} S(t+1-r)(f(\alpha, X(r))-u(r)) d r \\
& +\int_{t}^{t+1} S(t+1-r) Q^{1 / 2} d W(r) \\
= & S(1) X(t)+\int_{0}^{1} S(1-r) \lambda(\alpha, r+t) d r+\int_{0}^{1} S(1-r) Q^{1 / 2} d \tilde{W}(r),
\end{aligned}
$$

where $\tilde{W}(r)=W(r+t)$ and

$$
\lambda(\alpha, s)=f(\alpha, X(s))-u(s) .
$$

Since it can be assumed that $\gamma \in(0,1)$ in $(\mathrm{A} 1)$, let $q=1 / \gamma$ and $v \in(1 / q, 1]$. Define the linear operator $J_{v}: L^{2}(0,1 ; H) \rightarrow H$ as

$$
J_{v} h=\int_{0}^{1}(1-s)^{v-1} S(1-s) h(s) d s .
$$

It is well known (e.g., [11]) that $J_{v}$ is a compact operator and

$$
\int_{0}^{1} S(1-r) Q^{1 / 2} d W(r)=\frac{\sin \frac{1}{2} \pi \gamma}{\pi} J_{\gamma / 2}(Y)
$$


where

$$
Y(s)=\int_{0}^{s}(s-u)^{-\gamma / 2} S(s-u) Q^{1 / 2} d W(u) .
$$

Thus (2.24) can be rewritten as

$$
X(t+1)=S(1) X(t)+J_{1} \lambda(\alpha, \cdot+t)+\frac{\sin \frac{1}{2} \pi \gamma}{\pi} J_{\gamma / 2}(Y)
$$

for $t \geq 0$. Let $|\cdot|_{q}$ be the norm in $L^{q}(0,1 ; H)$. By (2.20) and (i) it follows that

$$
\mathbb{E}_{x, u}^{\alpha}|\lambda(\alpha, \cdot+t)|_{q}^{q} \leq \mathbb{E}_{x, u}^{\alpha} k_{1}\left(R^{q}+k_{2} \int_{0}^{1}|X(r+t)|^{q p} d t\right) \leq k_{3}
$$

and (A1) implies that

$$
\mathbb{E}_{x, u}^{\alpha} \int_{0}^{1}|Y(s)|^{2} d s \leq k_{4} \int_{0}^{1}\left(\int_{0}^{s}(s-u)^{-\gamma}|S(s-u)|_{\mathrm{HS}}^{2} d u\right)^{2} d s \leq k_{5}
$$

for some constants $k_{1}-k_{5}$ that do not depend on $t \in \mathbb{R}_{+}, \alpha \in \mathcal{A}$, and $u \in \mathcal{U}$. Define a sequence of compact sets $\left(K_{n}, n \in \mathbb{N}\right)$ as

$$
K_{n}=\left\{\left.y \in H\left|y \in S(1) x+J_{1} h+J_{\gamma / 2} g,\right| x\right|^{2}+|h|_{q}^{q}+|g|_{q}^{q} \leq n\right\} .
$$

By the Chebyshev inequality it follows that

$$
\mathbb{P}_{x, u}^{\alpha}\left(X(t+1) \notin K_{n}\right) \leq \frac{1}{n}\left[\mathbb{E}_{x, u}^{\alpha}\left(|X(t)|^{2}+|\lambda(\alpha, \cdot+t)|_{q}^{q}+\left(\frac{\sin \frac{1}{2} \pi \gamma}{\pi}\right)^{q}|Y|_{q}^{q}\right)\right]
$$

so that

$$
\lim _{n \rightarrow \infty} \sup _{t \geq 1} \mathbb{P}_{x, u}^{\alpha}\left(X(t) \in H \backslash K_{n}\right)=0
$$

by $(2.25),(2.26)$, and (i).

In what follows let $\left(P_{t}^{\alpha}, t \geq 0\right)$ be the Markov transition semigroup induced by the solution of (2.1) with $u \equiv 0$. It is clear that for each $\alpha \in \mathcal{A},\left(P_{t}^{\alpha}, t \geq 0\right)$ is a semigroup of bounded, linear operators on $C_{b}(H)$.

This section is concluded with some bounds on the Fréchet derivative of $P_{t}^{\alpha} \varphi$ for $t \geq 0$. For a bounded, Borel measurable function $\phi: H \rightarrow \mathbb{R}$, let $\|\phi\|=\sup _{x \in H}|\phi(x)|$ be the essential supremum.

Proposition 2.2. If (A1) and (A2) are satisfied with $\omega-\beta>0$, then for each $t>0, \alpha \in \mathcal{A}$, and $\varphi \in C_{b}(H)$ the function $P_{t}^{\alpha} \varphi: H \rightarrow C_{b}(H)$ is Fréchet differentiable and its Fréchet derivative $D P_{t}^{\alpha} \varphi$ satisfies the following inequalities:

$$
\left\|D P_{t}^{\alpha} \varphi\right\| \leq\left|Q^{-1 / 2}\right|_{\mathcal{L}(H)} t^{-1 / 2}\|\varphi\|, \quad t>0,
$$

and

$$
\left\|D P_{t}^{\alpha} \varphi\right\| \leq\left|Q^{-1 / 2}\right|_{\mathcal{L}(H)} e^{-(\omega-\beta)(t-1)}\|\varphi\|, \quad t>1,
$$

where $\|\cdot\|=\sup _{x \in H}|\cdot|$. From (2.27) and (2.28) the following inequality is satisfied:

$$
\left\|D P_{t}^{\alpha} \varphi\right\| \leq k\left(\omega_{1}\right)\left|Q^{-1 / 2}\right|_{\mathcal{L}(H)} t^{-1 / 2} e^{-\omega_{1} t}\|\varphi\|
$$


for $t>0$ and each $\omega_{1} \in(0, \omega-\beta)$ for a constant $k\left(\omega_{1}\right)$ that depends only on $\omega_{1}, \omega$, and $\beta$.

Proof. Let $\left(X^{\alpha, x}(t), t \geq 0\right)$ be the solution of (2.1) with $u \equiv 0$. By Proposition 7 in [10] it follows that the map $x \mapsto X^{\alpha, x}(t)$ is Gâteaux differentiable in the mean square for each $t \in \mathbb{R}_{+}$and its directional derivative at $x$ in the direction $h \in H$, $Y_{h}^{\alpha, x}(t)$, is a mild solution of the random linear differential equation

$$
\begin{aligned}
\frac{d}{d t} Y_{h}^{\alpha, x}(t) & =\left(A+D f\left(\alpha, X^{\alpha, x}(t)\right)\right) Y_{h}^{\alpha, x}(t), \\
Y_{h}^{\alpha, x}(0) & =h .
\end{aligned}
$$

Let $g_{n}(\alpha, x)=n(n I-A)^{-1} D f(\alpha, x)$ for $n \in \mathbb{N}$ and let $\left(y_{n}(t), t \geq 0\right)$ be the strong solution of the random linear differential equation

$$
\begin{aligned}
\frac{d}{d t} y_{n}(t) & =\left(A+g_{n}\left(\alpha, X^{\alpha, x}(t)\right)\right) y_{n}(t), \\
y_{n}(0) & =n(n I-A)^{-1} h=h_{n} .
\end{aligned}
$$

By (A1) it follows that

$$
\begin{aligned}
\frac{d}{d t}\left|y_{n}(t)\right|^{2} & =2\left\langle\left(A+g_{n}\left(\alpha, X^{\alpha, x}(t)\right)\right) y_{n}(t), y_{n}(t)\right\rangle \\
& \leq-2 \omega\left|y_{n}(t)\right|^{2}+2\left\langle g_{n}\left(\alpha, X^{\alpha, x}(t)\right) y_{n}(t), y_{n}(t)\right\rangle
\end{aligned}
$$

so that

$$
\left|y_{n}(t)\right|^{2} \leq\left|h_{n}\right|^{2}-2 \omega \int_{0}^{t}\left|y_{n}(s)\right|^{2} d s+2 \int_{0}^{t}\left\langle g_{n}\left(\alpha, X^{\alpha, x}(s)\right) y_{n}(s), y_{n}(s)\right\rangle d s .
$$

Letting $n \rightarrow \infty$ and using standard properties of the Yoshida approximations and (A2) it follows that

$$
\left|Y_{k}^{\alpha, x}(t)\right| \leq|h|^{2}-2(\omega-\beta) \int_{0}^{t}\left|Y_{h}^{\alpha, x}(s)\right|^{2} d s
$$

and

$$
\left|Y_{h}^{\alpha, x}(t)\right|^{2} \leq|h|^{2} e^{-2(\omega-\beta) t} .
$$

By Theorem 4.1 in [12] it follows that $D P_{t}^{\alpha} \varphi \in C_{b}(H)$ for $t>0$ and

$$
\left\langle D P_{t}^{\alpha} \varphi(x), h\right\rangle=\frac{1}{t} \mathbb{E} \varphi\left(X^{\alpha, x}(t)\right) \int_{0}^{t}\left\langle Q^{-1 / 2} Y_{h}^{\alpha, x}(s), d W(s)\right\rangle
$$

for $t>0$ is satisfied for each $h \in H$, so by (2.30) it follows that

$$
\left\|D P_{t}^{\alpha} \varphi\right\| \leq\left|Q^{-1 / 2}\right|_{\mathcal{L}(H)} t^{-1 / 2}\|\varphi\|
$$

for $t>0$ and (2.27) is verified. Furthermore, setting $\varphi_{1}^{\alpha}=P_{1}^{\alpha} \varphi$ and using the semigroup property of $P_{t}^{\alpha}$ it follows that

$$
\begin{aligned}
\left\langle D P_{t}^{\alpha} \varphi(x), h\right\rangle & =\left\langle D P_{t-1}^{\alpha} \varphi_{1}^{\alpha}(x), h\right\rangle \\
& =\left\langle D\left(\mathbb{E} \varphi_{1}^{\alpha}\left(X^{\alpha, x}(t-1)\right), h\right\rangle\right. \\
& =\mathbb{E}\left\langle D \varphi_{1}^{\alpha}\left(X^{\alpha, u}(t-1), Y_{h}^{\alpha, x}(t-1)\right\rangle\right.
\end{aligned}
$$

for $t>1, h \in H$, and $x \in H$. Now using (2.30) and (2.31) with $t=1$ it follows that

$$
\left\|D P_{t}^{\alpha} \varphi\right\| \leq\left|Q^{-1 / 2}\right|_{\mathcal{L}(H)}\|\varphi\| e^{-(\omega-\beta)(t-1)}
$$

for $t \geq 1$. 
3. Parameter estimation. In this section the estimation of the unknown parameter is considered where the parameter appears affinely in $f$, that is,

$$
f(\alpha, x)=f_{o}(x)+\sum_{i=1}^{q} \alpha^{i} f_{i}(x)
$$

where $\alpha=\left(\alpha^{1}, \ldots, \alpha^{q}\right)^{\mathrm{T}}$. It is assumed that $f_{0}, f_{1}, \ldots, f_{q}$ satisfy the relevant conditions on $f$ in (A2) and (A3).

Let $(u(t), t \geq 0)$ be an admissible control and let $(X(t), t \geq 0)$ be the associated solution of (2.1). For notational simplicity, the dependence of $X$ on $u$ is suppressed. Let

$$
\mathcal{A}(t)=\left(a_{i j}(t)\right)
$$

and

$$
\tilde{\mathcal{A}}(t)=\left(\tilde{a}_{i j}(t)\right)
$$

for $t \geq 0$ be two $\mathcal{L}\left(\mathbb{R}^{q}, \mathbb{R}^{q}\right)$-valued processes, where

$$
a_{i j}(t)=\int_{0}^{t}\left\langle P f_{i}(X(s)), P f_{j}(X(s))\right\rangle d s
$$

and

$$
\tilde{a}_{i j}(t)=\frac{a_{i j}(t)}{a_{i i}(t)}
$$

and $P: H \rightarrow P(H)$ is a fixed finite dimensional projection on $H$ with range in $\operatorname{Dom}\left(A^{*}\right)$ that is chosen to satisfy the subsequent assumptions (A6) and (A7).

For the verification of the strong consistency (e.g., [26]) of a family of least squares estimates of the unknown parameter vector, the following two assumptions are used.

(A6) For each admissible control law, the $\mathcal{L}\left(\mathbb{R}^{q}, \mathbb{R}^{q}\right)$-valued process $(\hat{\mathcal{A}}(t), t \geq 0)$ satisfies

$$
\liminf _{t \rightarrow \infty}|\operatorname{det} \tilde{\mathcal{A}}(t)|>0 \quad \text { a.s. }
$$

and

(A7) there is a $c>0$ such that $\left|P f_{i}(x)\right|^{2}>c$ for $i \in\{1, \ldots, q\}$ and all $x \in H$.

It is elementary to give examples where (A6) and (A7) are satisfied. For example, (A6) and (A7) are trivially satisfied if $\left(P f_{1}, P f_{2}, \ldots, P f_{q}\right)$ are nonzero, orthogonal elements for each $x \in H$ and their norms are uniformly bounded away from zero.

The estimate of the unknown parameter vector at time $t, \hat{\alpha}(t)$, is the minimizer of the quadratic functional of $\alpha, L(t ; \alpha)$, given by

$$
\begin{aligned}
L(t ; \alpha)= & -\int_{0}^{t} \sum_{i=1}^{q}\left\langle P \alpha^{i} f_{i}(X(s)), d P X(s)\right\rangle \\
& +\frac{1}{2} \int_{0}^{t} \sum_{i=1}^{q}\left|P \alpha^{i} f_{i}(X(s))\right|^{2} d s .
\end{aligned}
$$


The minimizer of (3.4) is the solution of the family of linear equations

$$
\mathcal{A}(t) \hat{\alpha}(t)=\mathcal{A}(t) \alpha_{0}+b(t)
$$

or equivalently

$$
\tilde{\mathcal{A}}(t) \hat{\alpha}(t)=\tilde{\mathcal{A}}(t) \alpha_{0}+\tilde{b}(t),
$$

where $\mathcal{A}(t)$ and $\tilde{\mathcal{A}}(t)$ are given by $(3.2)$ and (3.3), respectively, $b(t)=\left(b_{1}(t), \ldots\right.$, $\left.b_{q}(t)\right)^{\mathrm{T}}, \tilde{b}(t)=\left(\tilde{b}_{1}(t), \ldots, \tilde{b}_{q}(t)\right)^{\mathrm{T}}$,

$$
b_{j}(t)=\int_{0}^{t}\left\langle P f_{i}(X(s)), d P Q^{1 / 2} W(t)\right\rangle
$$

and

$$
\tilde{b}_{j}(t)=\frac{b_{j}(t)}{a_{j j}(t)}
$$

for $j=\{1, \ldots, q\}$, and $\alpha_{0}$ is the true parameter vector.

The family of estimates $(\hat{\alpha}(t), t \geq 0)$ is strongly consistent as described in the following result.

TheOREM 3.1. Let $(u(t), t \geq 0)$ be an admissible control law. If (A1)-(A4) and (A6)-(A7) are satisfied, then the family of least squares estimates $(\hat{\alpha}(t), t \geq 0)$, where $\hat{\alpha}(t)$ is the solution of (3.6), is strongly consistent, that is,

$$
\lim _{t \rightarrow \infty} \hat{\alpha}(t)=\alpha_{0} \quad \text { a.s. },
$$

where $\alpha_{0}$ is the true parameter vector.

Proof. By (A7), a time change in the stochastic integrals in the components of $b(t)$, and the law of large numbers for Brownian motion, it follows that

$$
\lim _{t \rightarrow \infty} \tilde{b}(t)=0 \quad \text { a.s. }
$$

The assumption (A6) ensures that for $t \gg 0 \tilde{\mathcal{A}}^{-1}(t, \omega)$ exists and is bounded for almost all $\omega$, so the equality (3.8) implies that $\hat{\alpha}(t) \rightarrow \alpha_{0}$ a.s. as $t \rightarrow \infty$.

In [21], the parameter estimation of $\alpha$ in (2.1) is considered where $f$ depends on $\alpha$ not necessarily affinely. With an identifiability condition and some other conditions, it is shown that a family of maximum likelihood estimates of $\alpha$ is strongly consistent. This result is a generalization of [5] to some infinite dimensional systems. The infinite dimensional setting presents some significant difficulties that either do not occur or are relatively easily overcome in finite dimensions, e.g., the application of an Itô formula without strong solutions, the tightness of a family of empirical measures, and some properties of Markov semigroups.

4. Adaptive control. In this section an adaptive control is constructed using a solution to the infinite dimensional HJB equation. This control is shown to be selfoptimizing for a strongly consistent family of estimates of the unknown parameter. To verify the self-optimality property, a continuous dependence of the solutions of the HJB equations with respect to the parameter in a suitable function space is an important tool. Initially the HJB equations are introduced corresponding to the discounted and the ergodic cost functionals (2.4) and (2.5) and a summary and some modifications of some known results on these control problems are given. 
The formal HJB equations corresponding to the control problems (2.1), (2.4) and (2.1), (2.5) are, respectively,

$$
\begin{aligned}
& \frac{1}{2} \operatorname{Tr} Q D^{2} v_{\alpha}^{\lambda}(x)+\left\langle A x, D v_{\alpha}^{\lambda}(x)\right\rangle+\left\langle f(\alpha, x), D v_{\alpha}^{\lambda}(x)\right\rangle \\
& \quad-\tilde{H}\left(D v_{\alpha}^{\lambda}(x)\right)+\psi(x)=\lambda v_{\alpha}^{\lambda}(x), \\
& \frac{1}{2} \operatorname{Tr} Q D^{2} v_{\alpha}(x)+\left\langle A x, D v_{\alpha}(x)\right\rangle+\left\langle f(\alpha, x), D v_{\alpha}(x)\right\rangle \\
& \quad-\tilde{H}\left(D v_{\alpha}(x)\right)+\psi(x)=\rho(\alpha) .
\end{aligned}
$$

In (4.2) it is necessary to solve for the pair $\left(v_{\alpha}, \rho(\alpha)\right), \rho(\alpha) \in \mathbb{R}$ for each $\alpha \in \mathcal{A}$.

The existence of strong solutions to (4.1) and (4.2) is unlikely because of the first two terms on the left-hand side of these equations, specifically because $Q$ is not trace class and $A$ is only densely defined in $H$. The approach in [20] is to replace the first two terms in (4.1) and (4.2) by the generator of an Ornstein-Uhlenbeck semigroup in a suitable function space. The results of [20], [22] are used but for simplicity the solutions of (4.1) and (4.2) are defined in a weaker sense which is suitable for the applications to adaptive control.

Let $\mu=N\left(0, Q_{\infty}\right)$ be the invariant measure and

$$
\left(R_{t} \varphi\right)(x)=\mathbb{E}_{x} \varphi(Z(t))
$$

be the Markov transition semigroup for the Ornstein-Uhlenbeck process $(Z(t), t \geq 0)$ that is the solution of $(2.6)$. It is well known that $\left(R_{t}, t \geq 0\right)$ is a strongly continuous semigroup on the Hilbert space

$$
\mathcal{H}=L^{2}(H, \mu) .
$$

Let $\mathcal{L}$ be the infinitesimal generator of the semigroup $\left(R_{t}, t \geq 0\right)$ in $\mathcal{H}$. Furthermore, let $\mathcal{L}_{0}$ be given by

$$
\mathcal{L}_{0} \varphi(x)=\frac{1}{2} \operatorname{Tr} Q D^{2} \varphi(x)+\left\langle x, A^{*} D \varphi(x)\right\rangle
$$

for $x \in H$ and $\varphi \in \operatorname{Dom}\left(\mathcal{L}_{0}\right)$, where $\operatorname{Dom}\left(\mathcal{L}_{0}\right)=\left\{\varphi \in C_{b}^{2}(H) \mid(1 / 2) \operatorname{Tr} Q D^{2} \varphi(\cdot) \in\right.$ $\left.C_{b}(H),\left\langle\cdot, A^{*} D \varphi(\cdot)\right\rangle \in C_{b}(H)\right\}$.

Let $\varphi \in \operatorname{Dom}\left(\mathcal{L}_{0}\right)$ and use the Itô formula to obtain

$$
\begin{aligned}
(\mathcal{L} \varphi)(x) & =\lim _{t \downarrow 0} \frac{1}{t}\left(R_{t} \varphi(x)-\varphi(x)\right) \\
& =\lim _{t \downarrow 0} \frac{1}{t}\left(\mathbb{E}_{x} \varphi(Z(t))-\varphi(x)\right) \\
& =\left(\mathcal{L}_{0} \varphi\right)(x)
\end{aligned}
$$

so $\mathcal{L}$ is a closed extension of the operator $\mathcal{L}_{0}$. This equality motivates the following definition of solution of (4.1) and (4.2).

Definition 4.1. A function $v_{\alpha}^{\lambda} \in \operatorname{Dom}(\mathcal{L})$ and a pair $\left(v_{\alpha}, \rho(\alpha)\right) \in \operatorname{Dom}(\mathcal{L}) \times \mathbb{R}$ are solutions to (4.1) and (4.2), respectively, if

$$
\mathcal{L} v_{\alpha}^{\lambda}+\left\langle f(\alpha, \cdot), D v_{\alpha}^{\lambda}\right\rangle-\tilde{H}\left(D v_{\alpha}^{\lambda}\right)+\psi=\lambda v_{\alpha}^{\lambda}
$$


and

$$
\mathcal{L} v_{\alpha}+\left\langle f(\alpha, \cdot), D v_{\alpha}\right\rangle-\tilde{H}\left(D v_{\alpha}\right)+\psi=\rho(\alpha)
$$

are satisfied.

This definition of solutions to (4.1) and (4.2) requires only that the solutions be in $\operatorname{Dom}(\mathcal{L}) \subset L^{2}(H, \mu)$ so (4.4) and (4.5) are understood in an $L^{2}(H, \mu)$ sense. This relatively weak notion of solution is used to avoid some technical complications. Some results on the solutions to (4.1) and (4.2) are given in [20] and [22]. It is shown that the solutions are more regular than that required in Definition 4.1. For the following two propositions the parameter $\alpha \in \mathcal{A}$ is fixed.

Proposition 4.1. If (A1), (A2), (A4), and (A5) are satisfied, then (4.1) has one and only one solution $v_{\alpha}^{\lambda}$ in $\operatorname{Dom}(\mathcal{L}) \cap C_{b}^{1}(H)$. Furthermore,

$$
v_{\alpha}^{\lambda}(x)=\inf _{u \in \mathcal{U}} J(x, \lambda, u)
$$

so that $v_{\alpha}^{\lambda}$ gives the optimal cost and an optimal control in feedback form is $\hat{u}_{\alpha}^{\lambda}(x)=$ $D \tilde{H}\left(D v_{\alpha}^{\lambda}(x)\right)$ for the discounted cost control problem $(2.1),(2.4)$.

This proposition has been basically proven by Gozzi and Rouy [22] when $f(\alpha, \cdot)$ is bounded. The generalization in Proposition 4.2 has been done by Goldys and Maslowski [20].

The ergodic control problem is usually considered to be more difficult than the discounted control problem because the HJB equation (4.2) has an intrinsic degeneracy; that is, there is no uniqueness of the solution to $(4.2)$ because if $\left(v_{\alpha}, \rho(\alpha)\right)$ is a solution of $(4.2)$, then $\left(v_{\alpha}+c, \rho(\alpha)\right)$ for $c \in \mathbb{R}$ is also a solution. The following proposition describes results in [20] for a slightly more general problem.

Proposition 4.2. If (A1), (A2), (A4), and (A5) are satisfied, where $\omega-\beta>0$ and

$$
R<\frac{\sqrt{\omega_{1}}}{\left|Q^{-1 / 2}\right|_{\mathcal{L}(H)} k\left(\omega_{1}\right) \sqrt{\pi}}
$$

where $\omega_{1} \in(0, \omega-\beta)$ and $k\left(\omega_{1}\right)>0$ is the constant given in $(2.29)$, then there is a unique solution $\left(v_{\alpha}, \rho(\alpha)\right) \in \operatorname{Dom}(\mathcal{L}) \times \mathbb{R}_{+}$such that $v_{\alpha} \in C^{1}(H), D v_{\alpha} \in C_{b}(H)$, and $v_{\alpha}(0)=0$. Furthermore,

$$
\rho(\alpha)=\inf _{u \in \mathcal{U}} \tilde{J}(x, u)
$$

so that $\rho(\alpha)$ is the optimal cost and an optimal control in feedback form is $\hat{u}_{\alpha}(x)=$ $D \tilde{H}\left(D v_{\alpha}(x)\right)$ for the ergodic control problem $(2.1),(2.5)$

The following result provides a relative compactness of some translates of $\left(v_{\alpha}^{\lambda}\right)$ that allows the ergodic solution $v_{\alpha}$ to be obtained as a "limit" of the discounted control problems.

Proposition 4.3. If (A1), (A2), (A4), and (A5) are satisfied, where $\omega-\beta>0$ and the inequality (4.7) are satisfied, then the family of functions $\left(\bar{v}_{\alpha}^{\lambda} ; \alpha \in \mathcal{A}, \lambda \in\right.$ $(0,1])$ is relatively compact in the Sobolev space $W^{1,2}(H, \mu)$, where $\bar{v}_{\alpha}^{\lambda}=v_{\alpha}^{\lambda}-c(\alpha, \lambda)$ and

$$
c(\alpha, \lambda)=\frac{1}{\lambda} \int_{H}\left(\left\langle f(\alpha, x), D v_{\alpha}^{\lambda}(x)\right\rangle-\tilde{H}\left(D v_{\alpha}^{\lambda}(x)\right)+\psi(x)\right) \mu(d x)
$$


Proof. Let $\hat{P}_{t}^{\alpha, \lambda}: C_{b}(H) \rightarrow C_{b}(H)$ be the Markov transition semigroup corresponding to the solution of (2.1) using the optimal control $\hat{u}_{\alpha}^{\lambda}$, that is, $\hat{P}_{t}^{\alpha, \lambda} \varphi(x)=$ $\mathbb{E} \varphi\left(X^{x, \alpha, \lambda}(t)\right)$, where $\left(X^{x, \alpha, \lambda}(t), t \geq 0\right)$ satisfies

$$
\begin{aligned}
d X^{x, \alpha, \lambda}(t) & =\left(A X^{x, \alpha, \lambda}(t)+f\left(\alpha, X^{x, \alpha, \lambda}(t)\right)-\hat{u}_{\alpha}^{\lambda}\left(X^{x, \alpha, \lambda}(t)\right)\right) d t+Q^{1 / 2} d W(t), \\
X^{x, \alpha, \lambda}(0) & =x
\end{aligned}
$$

Initially it is verified that there is a function $\gamma \in L^{1}(0, \infty)$ that does not depend on $\alpha$ or $\lambda$ such that

$$
\left\|D \hat{P}_{t}^{\alpha, \lambda} \varphi\right\| \leq \gamma(t)\|\varphi\|
$$

for each $\varphi \in C_{b}(H)$. It is known that the function $\xi(t, x)=\hat{P}_{t}^{\alpha, \lambda} \varphi(x)$ is a solution to the backward Kolmogorov equation, which is defined as a mild solution, that is, $\xi(t, \cdot) \in C_{b}^{1}(H)$ for each $t>0$ and it satisfies the integral equation

$$
\xi(t, x)=P_{t}^{\alpha} \varphi(x)+\int_{0}^{t} P_{t-s}^{\alpha}\left\langle-\hat{u}_{\alpha}^{\lambda}(\cdot), D \xi(s, \cdot)\right\rangle(x) d s
$$

for $t>0$, where $\left(P_{t}^{\alpha}, t \geq 0\right)$ is the Markov transition semigroup of the solution of (2.1) with $u \equiv 0$. Using the differentiability of (4.10) in $x$ and Proposition 2.2, it follows that

$$
\begin{aligned}
\left\|D \hat{P}_{t}^{\alpha, \lambda} \varphi\right\| \leq & k\left(\omega_{1}\right)\left|Q^{-1 / 2}\right|_{\mathcal{L}(H)} t^{-1 / 2} e^{-\omega_{1} t}\|\varphi\| \\
& +\int_{0}^{t} k\left(\omega_{1}\right)\left|Q^{-1 / 2}\right|_{\mathcal{L}(H)}(t-s)^{-1 / 2} e^{-\omega_{1}(t-s)} R\left\|D \hat{P}_{s}^{\alpha, \lambda} \varphi\right\| d s
\end{aligned}
$$

for $t>0$, where $\omega_{1} \in(0, \omega-\beta)$ and $k\left(\omega_{1}\right)$ is given in $(2.29)$. Let $c=k\left(\omega_{1}\right)\left|Q^{-1 / 2}\right|_{\mathcal{L}(H)}\|\varphi\|$ and $C=k\left(\omega_{1}\right)\left|Q^{-1 / 2}\right|_{\mathcal{L}(H)} R$ so (4.11) can be written as

$$
\left\|D \hat{P}_{t}^{\alpha, \lambda} \varphi\right\| \leq c t^{-1 / 2} e^{-\omega_{1} t}+C \int_{0}^{t}(t-s)^{-1 / 2} e^{-\omega_{1}(t-s)}\left\|D \hat{P}_{t}^{\alpha, \lambda} \varphi\right\| d s .
$$

By the generalized Gronwall lemma (Lemma 7.11 in [24]), if

$$
\left(C \Gamma\left(\frac{1}{2}\right)\right)^{2}<\theta<\omega_{1},
$$

where $\Gamma(\cdot)$ is the gamma function, then there is a universal constant $k>0$ such that

$$
\left\|D \hat{P}_{t}^{\alpha, \lambda} \varphi\right\| \leq \gamma(t)\|\varphi\|
$$

for $t>0$, where

$$
\begin{aligned}
\gamma(t)= & k\left(\omega_{1}\right)\left|Q^{-1 / 2}\right|_{\mathcal{L}(H)} \\
& \times\left(e^{-\omega_{1} t} t^{-1 / 2}+k \int_{0}^{t} e^{\left(\theta-\omega_{1}\right)(t-s)}(t-s)^{-1 / 2} e^{-\omega_{1} s} s^{-1 / 2} d s\right)
\end{aligned}
$$

and $\gamma \in L^{1}(0, \infty)$ by a property of convolution from Young's inequality. The inequality (4.7) guarantees that (4.12) is satisfied for some $\theta$ so the inequality (4.9) is satisfied. 
Next it is shown that there is a constant $C_{1}$ that does not depend on $\alpha$ or $\lambda$ such that

$$
\left\|D \bar{v}_{\alpha}^{\lambda}\right\| \leq C_{1}
$$

for $\lambda \in(0,1]$ and $\alpha \in \mathcal{A}$. Since $v_{\alpha}^{\lambda}$ is the optimal cost and $\hat{u}_{\alpha}^{\lambda}$ is the optimal control for the discounted control problem (2.1), (2.4) from Proposition 2.2, it follows that

$$
v_{\alpha}^{\lambda}(x)=\int_{0}^{\infty} e^{-\lambda t} \hat{P}_{t}^{\alpha, \lambda}\left(\psi+h\left(\hat{u}_{\lambda}^{\alpha}\right)\right)(x) d t
$$

for each $x \in H, \lambda \in(0,1]$, and $\alpha \in \mathcal{A}$. Since $v_{\alpha}^{\lambda}$ and $\bar{v}_{\alpha}^{\lambda}$ differ only by a constant, it follows from (4.9) that

$$
\begin{aligned}
\left\|D \bar{v}_{\alpha}^{\lambda}\right\| & \leq \int_{0}^{\infty}\left\|D \hat{P}_{t}^{\alpha, \lambda}\left(\psi+h\left(\hat{u}_{\lambda}^{\alpha}\right)\right)\right\| d t \\
& \leq\left(\|\varphi\|+\sup _{|y| \leq R}|h(y)|\right) \int_{0}^{\infty} \gamma(t) d t:=C_{1} .
\end{aligned}
$$

Let $Q_{t}=\int_{0}^{t} S(r) Q S^{*}(r) d r$. Clearly $Q_{t}$ is the covariance operator of the (Gaussian) probability law of $Z(t)$, where $(Z(t), t \geq 0)$ is the Ornstein-Uhlenbeck process that satisfies (2.6). It is known that $(Z(t), t \geq 0)$ is strongly Feller, that is, Range $(S(t)) \subset$ Range $\left(Q_{t}^{1 / 2}\right)$ for $t>0$ so the operator $\Gamma(t)=Q_{t}^{-1 / 2} S(t) \in \mathcal{L}(H)$ [11]. Furthermore, it follows from $[8]$ and $[20]$ that

$$
\int_{0}^{\infty}|\Gamma(t)|_{\mathcal{L}(H)} d t<\infty .
$$

The resolvent of the infinitesimal generator $\mathcal{L}$ can be expressed as

$$
(\lambda I-\mathcal{L})^{-1} \varphi=\int_{0}^{\infty} e^{-\lambda t} R_{t} \varphi d t
$$

for $\varphi \in \mathcal{H}$ so the solution $v_{\alpha}^{\lambda}$ of (4.4) satisfies the integral equation

$$
v_{\alpha}^{\lambda}=\int_{0}^{\infty} e^{-\lambda t} R_{t}\left(\left\langle f(\alpha, \cdot), D v_{\alpha}^{\lambda}\right\rangle-\tilde{H}\left(D v_{\alpha}^{\lambda}\right)+\psi\right) d t .
$$

Since the inequality

$$
\left|D R_{t} \varphi\right|_{L^{2}(H, \mu ; H)} \leq|\Gamma(t)|_{\mathcal{L}(H)}|\varphi|_{\mathcal{H}}
$$

is satisfied for $t>0$, by the Cameron-Martin formula (Lemma 3 in [7]) the integral operators

$$
T_{\lambda}=\int_{0}^{\infty} e^{-\lambda t} D R_{t} d t
$$

for $\lambda \in[0,1]$ converge in the $\mathcal{L}\left(\mathcal{H}, L^{2}(H, \mu ; H)\right)$ norm. Since for each $t>0$ the linear operator $D R_{t}: \mathcal{H} \rightarrow L^{2}(H, \mu ; H)$ is compact [7], the operators $T_{\lambda}$ are also compact. Thus

$$
D \bar{v}_{\alpha}^{\lambda}=D v_{\alpha}^{\lambda}=T_{\lambda} \xi_{\alpha}^{\lambda}
$$


where $T$. : $[0,1] \rightarrow \mathcal{L}\left(\mathcal{H}, L^{2}(H, \mu ; H)\right)$ is continuous and

$$
\xi_{\alpha}^{\lambda}:=\left\langle f(\alpha, \cdot), D v_{\alpha}^{\lambda}\right\rangle-\tilde{H}\left(D v_{\alpha}^{\lambda}\right)+\psi
$$

for $\alpha \in \mathcal{A}$ and $\lambda \in(0,1]$ are uniformly bounded in $\mathcal{H}$ by (4.13). It follows that the family $\left(D \bar{v}_{\alpha}^{\lambda}, \alpha \in \mathcal{A}, \lambda \in(0,1]\right)$ is relatively compact in $L^{2}(H, \mu ; H)$. Note that

$$
\bar{v}_{\alpha}^{\lambda}=\int_{0}^{\infty} e^{-\lambda t} R_{t}\left(\xi_{\alpha}^{\lambda}-\lambda c(\lambda, \alpha)\right) d t,
$$

where

$$
\int_{H}\left(\xi_{\alpha}^{\lambda}-\lambda c(\lambda, \alpha)\right) d \mu=0 .
$$

Furthermore, by [7] the semigroup is compact in $\mathcal{H}$ and there is a $\lambda_{1}>0$ such that $\left|R_{t} \varphi\right|_{\mathcal{H}} \leq e^{-\lambda_{1} t}|\varphi|_{\mathcal{H}}$ for each $\varphi \in \mathcal{H}$ satisfying $\int \varphi d \mu=0$. Using (4.16) and (4.17), $v_{\alpha}^{\lambda}$ can replace $D \bar{v}_{\alpha}^{\lambda}$ above to verify that $\left(v_{\alpha}^{\lambda}, \alpha \in \mathcal{A}, \lambda \in(0,1]\right)$ is relatively compact in $\mathcal{H}$.

A result on the approximation of functions in $\operatorname{Dom}(\mathcal{L})$ by functions in $\operatorname{Dom}\left(\mathcal{L}_{0}\right)$ is given. Similar results in different spaces have been given (e.g., [6], [20], [22]). Let $\mathcal{W} \subset W^{1,2}(H, \mu)$ be given by

$$
\begin{gathered}
\mathcal{W}=\left\{\varphi \in W^{1,2}(H, \mu) \mid \varphi \in \operatorname{Dom}(\mathcal{L}),\|D \varphi\|<\infty,\right. \\
\qquad|\varphi(x)|+|\mathcal{L} \varphi(x)| \leq k\left(1+|x|^{q}\right) \text { for all } x \in H \\
\text { and some real numbers } k \text { and } q\} .
\end{gathered}
$$

Lemma 4.1. If $\varphi \in \mathcal{W}$, then there is a sequence $\left(\varphi_{n}, n \in \mathbb{N}\right)$ such that $\varphi_{n} \in$ $\operatorname{Dom}\left(\mathcal{L}_{0}\right), \varphi_{n} \in \mathcal{W}$ for fixed $k$ and $q$ defining $\mathcal{W}$ for all $n \in \mathbb{N}, \sup _{n}\left\|D \varphi_{n}\right\|<\infty$, and

$$
\begin{aligned}
\lim _{n \rightarrow \infty}\left|\varphi_{n}-\varphi\right|_{\mathcal{H}} & =0, \\
\lim _{n \rightarrow \infty}\left|\mathcal{L}_{0} \varphi_{n}-\mathcal{L} \varphi\right|_{\mathcal{H}} & =0, \\
\lim _{n \rightarrow \infty}\left|D \varphi_{n}-D \varphi\right|_{L^{2}(H, \mu ; H)} & =0 .
\end{aligned}
$$

Proof. Choose $\lambda>0$ and fix it. Let $\xi=\lambda \varphi-\mathcal{L} \varphi$. Since the inequality

$$
|\xi(x)| \leq k\left(1+|x|^{q}\right)
$$

for all $x \in H$ is satisfied, there is a sequence $\left(\xi_{n}, n \in \mathbb{N}\right)$ such that $\xi_{n} \in \operatorname{Dom}\left(\mathcal{L}_{0}\right)$ and $\xi_{n}$ satisfies (4.21) for all $n \in \mathbb{N}, \xi_{n} \rightarrow \xi$ in $\mathcal{H}$ as $n \rightarrow \infty$, and $\sup _{n}\left\|D \xi_{n}\right\|<\infty$. A similar construction is given in Lemma 4.5 in [20]. Now let

$$
\varphi_{n}=\int_{0}^{\infty} e^{-\lambda t} R_{t} \xi_{n} d t .
$$

Since $(\lambda I-\mathcal{L})^{-1}: \operatorname{Dom}\left(\mathcal{L}_{0}\right) \rightarrow \operatorname{Dom}\left(\mathcal{L}_{0}\right)$ and $\varphi_{n}=(\lambda I-\mathcal{L})^{-1} \xi_{n}$, it follows that $\varphi_{n} \in \operatorname{Dom}\left(\mathcal{L}_{0}\right)$. Furthermore,

$$
\left|\varphi_{n}-\varphi\right|_{\mathcal{H}} \leq \int_{0}^{\infty} e^{-\lambda t}\left|R_{t}\left(\xi_{n}-\xi\right)\right|_{\mathcal{H}} d t \leq\left|\xi_{n}-\xi\right|_{\mathcal{H}} \int_{0}^{\infty} e^{-\lambda t} d t
$$


and

$$
\begin{aligned}
\left|D \varphi_{n}-D \varphi\right|_{L^{2}(H, \mu ; H)} & \leq \int_{0}^{\infty} e^{-\lambda t}\left|D R_{t}\left(\xi_{n}-\xi\right)\right|_{L^{2}(H, \mu ; H)} d t \\
& \leq\left|\xi_{n}-\xi\right|_{\mathcal{H}} \int_{0}^{\infty} e^{-\lambda t}|\Gamma(t)|_{\mathcal{L}(H)} d t .
\end{aligned}
$$

This latter inequality follows from (4.15) and the convergence of the right-hand side to zero as $n \rightarrow \infty$ follows from (4.14) and the definition of $\left(\xi_{n}, n \in \mathbb{N}\right)$. It can be verified directly that

$$
\left\|D R_{t} \xi_{n}\right\|=\left\|D \mathbb{E} \xi_{n}\left(S(t) x+\int_{0}^{t} S(t-r) Q^{1 / 2} d W(r)\right)\right\| \leq\left\|D \xi_{n}\right\|
$$

for $t>0$ so that

$$
\sup _{n}\left\|D \varphi_{n}\right\| \leq \frac{1}{\lambda} \sup _{n}\left\|D \xi_{n}\right\|<\infty .
$$

Since $\mathcal{L}_{0} \varphi_{n}=\lambda \varphi_{n}-\xi_{n}$ it follows that $\mathcal{L}_{0} \varphi_{n}=\mathcal{L} \varphi_{n} \rightarrow y$ on $\mathcal{H}$ as $n \rightarrow \infty$ for some $y \in \mathcal{H}$ because $\mathcal{L}$ is a closed operator. Thus, $y=\mathcal{L} \varphi$. The uniform polynomial bounds on $\varphi_{n}$ and thereby on $\mathcal{L}_{0} \varphi_{n}$ follow from the same bounds on $\xi_{n}$ and (4.22).

The following proposition is a " $W^{1,2}(H, \mu)$-version" of a result in [20] on the existence and the uniqueness of a solution to the ergodic HJB equation which is described in Proposition 4.2. While the existence result is weaker than the one in Proposition 4.2, the family of solutions for uniqueness is enlarged. The parameter $\alpha \in \mathcal{A}$ in the following proposition is fixed.

Proposition 4.4. If (A1), (A2), (A4), (A5) with $\omega-\beta>0$, and (4.7) are satisfied, then there is a unique solution $\left(v_{\alpha}, \rho(\alpha)\right) \in(\mathcal{W} \cap C(H)) \times \mathbb{R}$ of $(4.2)$ such that $v_{\alpha}(0)=0$. Furthermore, the equality (4.8) is satisfied and an optimal control in feedback form is $\hat{u}_{\alpha}(x)=D \tilde{H}\left(D v_{\alpha}(x)\right)$ for the ergodic control problem (2.1), (2.5).

Proof. The existence of the solution $\left(v_{\alpha}, \rho(\alpha)\right)$ with the required properties including (4.8) and an optimal feedback control follow from a result in [20] that is given here as Proposition 4.2. However, it should be noted that the existence of a solution is a simple consequence of Proposition 4.3. Since $(|\lambda c(\alpha, \lambda)|, \lambda \in(0,1])$ is uniformly bounded, there is a sequence $\left(\lambda_{n}, n \in \mathbb{N}\right)$ such that $\lambda_{n} \downarrow 0$ and $\left(\bar{v}_{\alpha}^{\lambda_{n}}, \lambda_{n} c\left(\alpha, \lambda_{n}\right)\right) \rightarrow$ $\left(\bar{v}_{\alpha}, \delta\right)$ in $W^{1,2}(H, \mu) \times \mathbb{R}$ for some $\left(\bar{v}_{\alpha}, \delta\right) \in W^{1,2}(H, \mu) \times \mathbb{R}$. Letting $\lambda_{n} \downarrow 0$ in (4.1) and using the closedness of $\mathcal{L}$ in $\mathcal{H}$ it follows that $\bar{v}_{\alpha} \in \operatorname{Dom}(\mathcal{L})$ and

$$
\mathcal{L} \bar{v}_{\alpha}+\left\langle f(\alpha, \cdot), D \bar{v}_{\alpha}\right\rangle-\tilde{H}\left(D \bar{v}_{\alpha}\right)+\psi=\delta
$$

is satisfied.

Now let $(\bar{v}, \bar{\delta}) \in(\mathcal{W} \cap C(H)) \times \mathbb{R}$ be a solution of (4.2) satisfying $\bar{v}(0)=0$. To verify uniqueness, it suffices to show that $(\bar{v}, \bar{\delta})=\left(v_{\alpha}, \rho(\alpha)\right)$, where $\left(v_{\alpha}, \rho(\alpha)\right)$ is the solution of (4.2) whose existence is given by Proposition 4.2.

Initially it is shown that

$$
\bar{\delta}=\rho(\alpha) .
$$

This verification is analogous to the corresponding part of the proof of Proposition 4.2 that is given in [20]. Let $\left(\bar{v}_{n}, n \in \mathbb{N}\right)$ be a sequence such that $\bar{v}_{n} \in \operatorname{Dom}\left(\mathcal{L}_{0}\right)$ for each $n \in \mathbb{N}$ and

$$
\bar{v}_{n} \rightarrow \bar{v}, \quad \mathcal{L}_{0} \bar{v}_{n} \rightarrow \mathcal{L} \bar{v} \quad \text { in } \mathcal{H},
$$




$$
\sup _{n}\left\|D \bar{v}_{n}\right\|<\infty, \quad D \bar{v}_{n} \rightarrow D \bar{v} \quad \text { in } L^{2}(H, \mu ; H),
$$

and $\bar{v}_{n}$ and $\mathcal{L}_{0} \bar{v}_{n}$ are uniformly, polynomially bounded (cf. Lemma 4.1). Clearly, the pair $\left(\bar{v}_{n}, \bar{\delta}\right)$ satisfies the equation

$$
\mathcal{L}_{0} \bar{v}_{n}+\left\langle f(\alpha, \cdot), D \bar{v}_{n}\right\rangle-\tilde{H}\left(D \bar{v}_{n}\right)+\psi_{n}=\bar{\delta},
$$

where

$$
\psi_{n}=\bar{\delta}+\tilde{H}\left(D \bar{v}_{n}\right)-\left\langle f(\alpha, \cdot), D \bar{v}_{n}\right\rangle-\mathcal{L}_{0} \bar{v}_{n} .
$$

Apply the Itô formula using the function $-\bar{\delta} t+\bar{v}_{n}(x)$ and the process that is the solution of (2.1) with the control $D \tilde{H}\left(D \bar{v}_{n}\right)$ to show that $\bar{\delta}$ is the optimal cost for the control problem (2.1) and (2.5), where $\psi$ is replaced by $\psi_{n}$ in (2.5). Since $\psi_{n} \rightarrow \psi$ (at least) pointwise by (4.24) and (4.25) and the sequence $\left(\psi_{n}, n \in \mathbb{N}\right)$ is uniformly polynomially bounded, the limit as $n \rightarrow \infty$ can be taken to show that $\bar{\delta}$ is the optimal cost for the control problem (2.1) and (2.5) so that (4.14) is verified.

It remains to show that $\bar{v}=v_{\alpha}$. Let $\left(v_{n}, n \in \mathbb{N}\right)$ be a sequence such that

$$
\begin{aligned}
v_{n} \rightarrow v_{\alpha}, \quad \mathcal{L}_{0} v_{n} \rightarrow \mathcal{L} v_{\alpha} & \text { in } \mathcal{H}, \\
\sup _{n}\left\|D v_{n}\right\|<\infty . \quad D v_{n} \rightarrow D v_{\alpha} & \text { in } L^{2}(H, \mu ; H),
\end{aligned}
$$

and $v_{n}$ and $\mathcal{L}_{0} v_{n}$ are uniformly, polynomially bounded using the notation of Lemma 4.1. Recall that $\alpha \in \mathcal{A}$ is fixed so the dependence of $v_{n}$ on $\alpha$ is suppressed. Let $\bar{u}_{n}=D \tilde{H}\left(D v_{n}\right)$ and $\bar{u}=D \tilde{H}\left(D v_{\alpha}\right)$ be controls. For an arbitrary $\Phi \in C([0, T], H)$ it easily follows from (4.27) that

$$
\begin{aligned}
& \left|\mathbb{E}_{\tau, x, \bar{u}_{n}} \Phi(X(\cdot))-\mathbb{E}_{\tau, x, \bar{u}} \Phi(X(\cdot))\right| \\
& \leq \mathbb{E}_{\tau, x} \Phi(X(\cdot)) \mid \exp \left(-\int_{0}^{T}\left\langle Q^{-1 / 2} \bar{u}_{n}(X(s)), d W(s)\right\rangle\right. \\
& \left.\quad-\frac{1}{2} \int_{0}^{T}\left|Q^{-1 / 2} \bar{u}_{n}(X(s))\right|^{2} d s\right) \\
& \quad-\exp \left(-\int_{0}^{T}\left\langle Q^{-1 / 2} \bar{u}(X(s)), d W(s)\right\rangle-\frac{1}{2} \int_{0}^{T}\left|Q^{-1 / 2} \bar{u}(X(s))\right|^{2} d s\right) \mid \\
& \rightarrow 0 \quad \text { as } n \rightarrow \infty
\end{aligned}
$$

for $\tau \in[0, T)$, where $\mathbb{E}_{\tau, x, u}$ and $\mathbb{E}_{\tau, x}$ denote the expectations associated with the initial condition $X(\tau)=x$ and $(X(t), t \geq \tau)$ is the solution to $(2.1)$ with $X(\tau)=x$ and $u \in \mathcal{U}$ and $u \equiv 0$, respectively. By the Skorokhod theorem there is a probability space $(\tilde{\Omega}, \tilde{\mathcal{F}}, \tilde{\mathbb{P}})$ and stochastic processes $\left(X_{n}(t), t \geq \tau\right)$ for $n \in \mathbb{N}$ and $\left(X_{0}(t), t \geq \tau\right)$ such that $X_{n}(\cdot)$ and $X_{0}(\cdot)$ have the probability laws on $C([\tau, \infty) ; H)$ that are identical with the solutions of (2.1) with the feedback controls $\bar{u}_{n}\left(X_{n}\right)$ and $\bar{u}\left(X_{0}\right)$, respectively, and

$$
\lim _{n \rightarrow \infty} \sup _{s \in[\tau, T]}\left|X_{n}(s)-X_{0}(s)\right|=0 \quad \text { a.s. } \tilde{\mathbb{P}}
$$


for each $T>\tau$. By the definition of $\tilde{H}$ in (A5) it follows that

$$
\begin{aligned}
0 & =\tilde{H}\left(D v_{\alpha}(x)\right)+h(\bar{u}(x))-\left\langle\bar{u}(x), D v_{\alpha}(x)\right\rangle \\
& \leq \tilde{H}(D \bar{v}(x))+h(\bar{u}(x))-\langle\bar{u}(x), D \bar{v}(x)\rangle
\end{aligned}
$$

so that

$$
\begin{aligned}
& \mathcal{L} v_{\alpha}(x)+\left\langle f(\alpha, x), D v_{\alpha}(x)\right\rangle-\left\langle\bar{u}(x), D v_{\alpha}(x)\right\rangle \\
& \quad \leq \mathcal{L} \bar{v}(x)+\langle f(\alpha, x), D \bar{v}(x)\rangle-\langle\bar{u}(x), D \bar{v}(x)\rangle,
\end{aligned}
$$

and therefore

$$
\begin{aligned}
& \mathcal{L} v_{n}(x)+\left\langle f(\alpha, x), D v_{n}(x)\right\rangle-\left\langle\bar{u}_{n}(x), D v_{n}(x)\right\rangle \\
& \quad \leq \mathcal{L}_{0} \bar{v}_{n}(x)+\left\langle f(\alpha, x), D \bar{v}_{n}(x)\right\rangle-\left\langle\bar{u}_{n}(x), D \bar{v}_{n}(x)\right\rangle+\delta_{n}(x)
\end{aligned}
$$

for all $x \in H$, where $\delta_{n}: H \rightarrow \mathbb{R}$ converges to 0 as $n \rightarrow \infty$ in $L^{2}(H, \mu)$ and $\left(\delta_{n}, n \in \mathbb{N}\right)$ is uniformly, polynomially bounded. Using the Itô formula it follows that the processes

$$
\Psi_{n}(t)=v_{n}\left(X_{n}(t)\right)-\bar{v}_{n}\left(X_{n}(t)\right)-\int_{\tau}^{t} \delta_{n}\left(X_{n}(s)\right) d s
$$

for $t \geq \tau$ satisfy the inequality

$$
\tilde{\mathbb{E}} \Psi_{n}(t) \leq \Psi_{n}(\tau)=v_{n}(x)-\bar{v}_{n}(x),
$$

where $\tilde{\mathbb{E}}$ is the expectation in $(\tilde{\Omega}, \tilde{\mathcal{F}}, \tilde{\mathbb{P}})$. Note that the probability laws for $X_{n}(s)$ for each $s \in \mathbb{R}_{+}$and $n \in \mathbb{N} \cup\{0\}$ are mutually absolutely continuous with $\mu$ because by the Girsanov theorem any of these measures is equivalent to the Gaussian measure $N\left(S(t) x, Q_{t}\right)$ (the law for $Z(t)$ from the solution of $(2.6)$ ) and $N\left(S(t) x, Q_{t}\right)$ is equivalent to $\mu$ from the strong Feller property for each $t>0$ and $x \in H$ (e.g., [27]). It follows that

$$
\lim _{n \rightarrow \infty} \tilde{\mathbb{E}} \Psi_{n}(t)=\tilde{\mathbb{E}}\left(v_{\alpha}\left(X_{0}(t)\right)-\bar{v}\left(X_{0}(t)\right)\right)
$$

and thus

$$
\mathbb{E}_{\tau, x, \bar{u}}\left(v_{\alpha}(X(t))-\bar{v}(X(t))\right) \leq v_{\alpha}(x)-\bar{v}(x)
$$

for $t \geq \tau$ and $x \in H$. Since $\tau \in[0, t]$ and $x \in H$ are arbitrary, it follows that the process $\left(v_{\alpha}(X(t))-\bar{v}(X(t)), t \geq 0\right)$, where $(X(t), t \geq 0)$ satisfies (2.1) with $u(t)=\bar{u}(X(t))$, is a supermartingale.

Furthermore,

$$
\sup _{t \geq 0} \mathbb{E}_{x, \bar{u}}\left|v_{\alpha}(X(t))-\bar{v}(X(t))\right| \leq k \sup _{t \geq 0} \mathbb{E}\left(1+|X(t)|^{q}\right)<\infty
$$

for some positive constants $k$ and $q$ by Proposition 2.1 (i). Thus, there is a limit of $\left(v_{\alpha}(X(t))-\bar{v}(X(t)), t \geq 0\right)$ a.s. $\quad \mathbb{P}_{x, \bar{u}}^{\alpha}$ as $t \rightarrow \infty$. Since the solution of (2.1) is ergodic for these feedback controls, for each ball $B$ in $H$ there is a sequence of random times $\left(\sigma_{n}, n \in \mathbb{N}\right)$ that increase to infinity such that $X\left(\sigma_{n}\right) \in B$ (cf. [29]). Since $v_{\alpha}(0)-\bar{v}(0)=0$ and $v_{\alpha}-\bar{v}: H \rightarrow \mathbb{R}$ is a continuous function, letting $B=B_{1 / n}$ for $n \in \mathbb{N}$ it follows that

$$
\liminf _{t \rightarrow \infty}\left(v_{\alpha}(X(t))-\bar{v}(X(t))\right)=0 \quad \text { a.s. } \mathbb{P}_{x, \bar{u}}^{\alpha}
$$


so that

$$
\lim _{t \rightarrow \infty}\left(v_{\alpha}(X(t))-\bar{v}(X(t))\right)=0 \quad \text { a.s. } \mathbb{P}_{x, \bar{u}}^{\alpha} .
$$

If there is a $y \in H$ such that $v_{\alpha}(y)-\bar{v}(y)>0$, then $v_{\alpha}-\bar{v}>\Delta>0$ in an open ball $B_{y}$ centered at $y$. Choosing $B=B_{y}$ above gives a contradiction to (4.28) so that $v_{\alpha} \equiv \bar{v}$.

Corollary 4.1. If (A1)-(A5) where $\omega-\beta>0$ are satisfied and (4.7) is satisfied, then the function $v .: \mathcal{A} \rightarrow W^{1,2}(H, \mu)$ given by $\alpha \mapsto v_{\alpha}$ is continuous and

$$
\left\|D v_{\alpha}\right\|<C_{1}
$$

for all $\alpha \in \mathcal{A}$ and some constant $C_{1}>0$ that does not depend on $\alpha \in \mathcal{A}$ where $v_{\alpha}$ is the unique solution of the ergodic HJB equation given in Proposition 4.4.

Proof. Let $\bar{v}_{\alpha}$ be the first component of the solution of the ergodic HJB which is given in the proof of Proposition 4.4 as a limit of $\bar{v}_{\alpha}^{\lambda}$ in $W^{1,2}(H, \mu)$ as $\lambda \downarrow 0$. By (4.13) it follows that

$$
\left\|D v_{\alpha}\right\|=\left\|D \bar{v}_{\alpha}\right\| \leq C_{1}
$$

because by the uniqueness part of Proposition $4.4 v_{\alpha}$ and $\bar{v}_{\alpha}$ differ only by a constant. Clearly the family $\left(\bar{v}_{\alpha}, \alpha \in \mathcal{A}\right)$ is relatively compact in $W^{1,2}(H, \mu)$ by Proposition 4.3. Using this relative compactness and the closedness of the derivative operator for any sequence $\left(\bar{v}_{\alpha_{n}}, n \in \mathbb{N}\right)$ such that $\alpha_{n} \rightarrow \alpha_{0}$ as $n \rightarrow \infty$ there is a subsequence again denoted $\left(\bar{v}_{\alpha_{n}}, n \in \mathbb{N}\right)$ such that $\bar{v}_{\alpha_{n}} \rightarrow \tilde{v}$ and $D \bar{v}_{\alpha_{n}} \rightarrow D \tilde{v}$ a.e. $\mu$ for some $\tilde{v}$. Furthermore, the sequence $\left(\rho\left(\alpha_{n}\right), n \in \mathbb{N}\right)$ is uniformly bounded by (4.13) and Proposition 4.4 and there is a subsequence again denoted by $\left(\rho\left(\alpha_{n}\right), n \in \mathbb{N}\right)$ such that $\rho\left(\alpha_{n}\right) \rightarrow \tilde{\rho}$ for some $\tilde{\rho} \in \mathbb{R}$. Since $\mathcal{L} \bar{v}_{\alpha_{n}}+\left\langle f\left(\alpha_{n}, \cdot\right), D \bar{v}_{n}\right\rangle-\tilde{H}\left(D \bar{v}_{\alpha_{n}}\right)+\psi=\rho\left(\alpha_{n}\right)$ and the operator $\mathcal{L}$ is closed in $\mathcal{H}$, it follows by (A3) that the limit as $n \rightarrow \infty$ is

$$
\mathcal{L} \tilde{v}+\langle f(\alpha, \cdot), D \tilde{v}\rangle-\tilde{H}(D \tilde{v})+\psi=\rho \quad \text { a.e. } \mu \text {. }
$$

By (4.29), $\tilde{v}$ is continuous and $D \tilde{v}$ is bounded so that $\tilde{v} \in \mathcal{W}$. By the uniqueness part of Proposition 4.4 it follows that $\tilde{v}-\tilde{v}(0)=v_{\alpha_{0}}$. By (4.28) and the $(\mu)$ almost sure convergence $\bar{v}_{\alpha_{n}} \rightarrow \tilde{v}$ as $n \rightarrow \infty$ it follows that $\bar{v}_{\alpha_{n}}(0) \rightarrow \tilde{v}(0)$. Therefore, $v_{\alpha_{n}}=\bar{v}_{\alpha_{n}}-\tilde{v}_{\alpha_{n}}(0) \rightarrow \tilde{v}-\tilde{v}(0)=v_{\alpha_{0}}$ as $n \rightarrow \infty$.

For a certainty equivalence adaptive control and a consistent family of estimators of the unknown parameter vector, it is shown that this control is self optimizing. Consider (2.1) with the true parameter value $\alpha_{0} \in \mathcal{A}$, that is,

$$
\begin{aligned}
d X(t) & =\left(A X(t)+f\left(\alpha_{0}, X(t)\right)-\tilde{u}(t)\right) d t+Q^{1 / 2} d W(t), \\
X(0) & =x,
\end{aligned}
$$

where

$$
\tilde{u}(t)=D \tilde{H}\left(D v_{\alpha(t)}(X(t))\right),
$$

where $(\alpha(t), t \geq 0)$ is an adapted, measurable, $\mathcal{A}$-valued process satisfying

$$
\lim _{t \rightarrow \infty} \alpha(t)=\alpha_{0}
$$

in probability $\mathbb{P}$ and $v_{\alpha}$ is given in Proposition 4.4. 
The following theorem and its corollary provide solutions to an adaptive control problem described by (2.1) and (2.5).

THEOREM 4.1. Given the adaptive control problem described by (2.1) and (2.5), where $\alpha_{0} \in \mathcal{A}$ is the true parameter vector, if (A1)-(A5) are satisfied, $\omega-\beta>0$, the inequality (4.7), is satisfied, and the $\mathcal{A}$-valued family of estimates of $\alpha_{0}(\alpha(t), t \geq 0)$, satisfies (4.31), then

$$
\tilde{J}(x, \tilde{u})=\rho\left(\alpha_{0}\right)
$$

for each $x \in H$, where $\tilde{u}$ is the adaptive control given by (4.30).

Proof. For notational simplicity, let $v=v_{\alpha_{0}}$ and $\rho=\rho\left(\alpha_{0}\right)$. Let $v_{n} \in \operatorname{Dom}\left(\mathcal{L}_{0}\right)$ for $n \in \mathbb{N}$ be a sequence that converges to $v$ as in (4.26), (4.27). The Itô formula applied to $\left(v_{n}(X(t))-\rho t, t \geq 0\right)$ yields the equation

$$
\begin{aligned}
&-\rho t+\mathbb{E}_{x, \tilde{u}} v_{n}(X(t)) \\
&=v_{n}(x)+\mathbb{E}_{x, \tilde{u}} \int_{0}^{t}\left[-\rho+\mathcal{L}_{0} v_{n}(X(s))+\left\langle f\left(\alpha_{0}, X(s)\right), D v_{n}(X(s))\right\rangle\right. \\
&\left.\quad-\left\langle\tilde{u}(s), D v_{n}(X(s))\right\rangle\right] d s .
\end{aligned}
$$

Since $v_{n}$ satisfies

$$
\mathcal{L}_{0} v_{n}+\left\langle f\left(\alpha_{0}, \cdot\right), D v_{n}\right\rangle-\tilde{H}\left(D v_{n}\right)+\psi_{n}=\rho
$$

where

$$
\psi_{n}=\rho+\tilde{H}\left(D v_{n}\right)-\left\langle f\left(\alpha_{0}, \cdot\right), D v_{n}\right\rangle-\mathcal{L}_{0} v_{n},
$$

it follows that

$$
\begin{aligned}
-\rho t & +\mathbb{E}_{x, \tilde{u}} v_{n}(X(t)) \\
& =v_{n}(x)+\mathbb{E}_{x, \tilde{u}} \int_{0}^{t}\left[-\psi_{n}(X(s))+\tilde{H}\left(D v_{n}(X(s))\right)-\left\langle\tilde{u}(s), D v_{n}(X(s))\right\rangle\right] d s .
\end{aligned}
$$

By the definition of $\tilde{H}$ in (A5) it follows that

$$
-\tilde{H}\left(D v_{n}(X(s))\right)-h(\tilde{u}(X(s)))+\left\langle\tilde{u}_{n}(X(s)), D v_{n}(X(s))\right\rangle=0,
$$

where

$$
\tilde{u}_{n}(x)=D \tilde{H}\left(D v_{n}(x)\right)
$$

Therefore,

$$
\begin{aligned}
\rho t= & \mathbb{E}_{x, \tilde{u}} v_{n}(X(t))-v_{n}(x) \\
& +\mathbb{E}_{x, \tilde{u}} \int_{0}^{t}\left[\psi_{n}(X(s))+h\left(\tilde{u}_{n}(X(s))\right)+\left\langle\tilde{u}(s)-\tilde{u}_{n}(X(s)), D v_{n}(X(s))\right\rangle\right] d s .
\end{aligned}
$$

Using the properties of convergence $v_{n} \rightarrow v$ in (4.26), (4.27), the uniform polynomial bound on the sequence $\left(v_{n}, n \in \mathbb{N}\right)$ and Proposition 2.1 (i), the passage to the limit $(n \rightarrow \infty)$ yields the equation

$$
\begin{aligned}
\rho t= & \mathbb{E}_{x, \tilde{u}} v(X(t))-v(x) \\
& \quad+\mathbb{E}_{x, \tilde{u}} \int_{0}^{t}\left[\psi(X(s))+h\left(\tilde{u}_{0}(X(s))\right)+\left\langle\tilde{u}(s)-\tilde{u}_{0}(X(s)), D v(X(s))\right\rangle\right] d s,
\end{aligned}
$$


where $\tilde{u}_{0}(x)=D \tilde{H}(D v(x))$. By Proposition 2.1 (i) it follows that

$$
\lim _{t \rightarrow \infty} \frac{1}{t} \mathbb{E}_{x, \tilde{u}} v(X(t))=0
$$

for each $x \in H$. By an interpolation result (Lemma 4.6 in [22]) it follows that

$$
\left\|D v_{\alpha}^{\lambda}\right\|_{r, \theta} \leq c_{1}+c_{2}\left\|D v_{\alpha}^{\lambda}\right\|+c_{3} \sup _{x \in B_{r}}|f(\alpha, x)|\left\|D v_{\alpha}^{\lambda}\right\|
$$

for some universal constants $c_{1}, c_{2}, c_{3}$ and a suitable constant $\theta>0$, where $\|\cdot\|_{r, \theta}$ denotes the norm of Hölder continuous functions with exponent $\theta$ on the ball $B_{r}$. The result in [22] is stated for $r=\infty$ and $f$ bounded but (4.34) follows directly if $f$ is truncated outside of $B_{r}$ and using the fact that $\mathcal{L}$ is a local operator so the truncation does not affect the solution $v_{\alpha}^{\lambda}$ in $B_{r}$. By (A3) and (4.13) it follows that $\left\|D v_{\alpha}^{\lambda}\right\|_{r, \theta} \leq c_{3}\left(1+r^{p}\right)$ for some constant $c_{3}$ that does not depend on $\alpha$ or $\lambda$. Thus by the proof of Corollary 4.1 it follows that

$$
\lim _{\alpha \rightarrow \alpha_{0}} D v_{\alpha}=D v_{\alpha_{0}}
$$

uniformly on compact sets. For a compact set $K \subset H$ if follows that

$$
\begin{aligned}
\mathbb{E}_{x, \tilde{u}}\left|D \tilde{H}\left(D v_{\alpha_{0}}(X(s))\right)-D \tilde{H}\left(D v_{\alpha(t)}(X(s))\right)\right| \\
=\mathbb{E}_{x, \tilde{u}}\left|D \tilde{H}\left(D v_{\alpha_{0}}(X(s))\right)-D \tilde{H}\left(D v_{\alpha(t)}(X(s))\right)\right| 1_{\{X(s) \in K\}} \\
\quad+\mathbb{E}_{x, \tilde{u}}\left|D \tilde{H}\left(D v_{\alpha_{0}}(X(s))\right)-D \tilde{H}\left(D v_{\alpha(t)}(X(s))\right)\right| 1_{\{X(s) \notin K\}} .
\end{aligned}
$$

The second term on the right-hand side of (4.36) is bounded above by

$$
2 R \mathbb{P}_{x, \tilde{u}}(X(s) \in H \backslash K)
$$

which can be made arbitrarily small for a compact set $K$ sufficiently large by Proposition 2.1 (iii) for $s \geq 1$ and $R$ is given in (2.2). The first term on the right-hand side of (4.36) tends to zero as $s \rightarrow \infty$ by the locally uniform convergence in (4.35). These two facts imply that

$$
\lim _{s \rightarrow \infty} \mathbb{E}_{x, \tilde{u}}\left|D \tilde{H}\left(D v_{\alpha_{0}}(X(s))\right)-D \tilde{H}\left(D v_{\alpha(s)}(X(s))\right)\right|=0 .
$$

By a similar argument it follows that

$$
\lim _{s \rightarrow \infty} \mathbb{E}_{x, \tilde{u}}\left|h\left(D \tilde{H}\left(D v_{\alpha_{0}}(X(s))\right)\right)-h\left(D \tilde{H}\left(D v_{\alpha(s)}(X(s))\right)\right)\right|=0 .
$$

Dividing (4.33) by $t$ and letting $t \rightarrow \infty$, the equality (4.32) follows by (4.37) and (4.38). This completes the proof. $\quad \square$

Corollary 4.2. Given the adaptive control problem described by (2.1) and (2.5), where $\alpha_{0} \in \mathcal{A}^{0}$, is the true parameter vector and $f$ satisfies (3.1). Let $(\alpha(t), t \geq 0)$ be the family of estimates of $\alpha_{0}$ given by

$$
\alpha(t)=1_{\{\hat{\alpha}(t) \in \mathcal{A}\}} \hat{\alpha}(t)+1_{\{\hat{\alpha}(t) \notin \mathcal{A}\}} \alpha^{*},
$$

where $\hat{\alpha}(t)$ is the solution of (3.5) and $\alpha^{*}$ is a fixed element of $\mathcal{A}$. If (A1)-(A7) are 
satisfied, $\omega-\beta>0$, and the inequality (4.7) is satisfied, then

$$
\tilde{J}(x, \tilde{u})=\rho\left(\alpha_{0}\right)
$$

for each $x \in H$, where $\tilde{u}$ is the adaptive control given by (4.30).

\section{REFERENCES}

[1] A. Bensoussan, Perturbation Methods in Optimal Control, John Wiley, New York, 1988.

[2] J. M. Bismut, Theorie probabiliste du controle des diffusion, Mem. Amer. Math. Soc., 167 (1976), pp. 1-130.

[3] V. S. Borkar, Optimal Control of Diffusion Processes, Longman Press, London, UK, 1989.

[4] V. S. Borkar and M. K. Ghosh, Ergodic control of multidimensional diffusions. I: The existence results, SIAM J. Control Optim., 26 (1988), pp. 112-126.

[5] V. S. Borkar and M. K. Ghosh, Ergodic control of multidimensional diffusions. II: Adaptive control, Appl. Math. Optim., 21 (1990), pp. 191-220.

[6] S. Cerrai And F. GozzI, Strong solutions of Cauchy problems associated to weakly continuous semigroups, Differential Integral Equations, 8 (1995), pp. 465-486.

[7] A. Chojnowska-Michalik And B. Goldys, Existence, uniqueness and invariant measures for stochastic semilinear equations in Hilbert spaces, Probab. Theory Related Fields, 102 (1995), pp. 331-356.

[8] A. Chojnowska-Michalik And B. Goldys, On regularity properties of nonsymmetric Ornstein-Uhlenbeck semigroup in $L^{p}$-spaces, Stochastics Stochastics Rep., 59 (1996), pp. 183-209.

[9] R. M. Cox, Stationary and Discounted Control Diffusion Processes, Ph.D. thesis, Columbia University, New York, 1984.

[10] G. Da Prato, K. D. Elworthy, and J. Zabczyk, Strong Feller property for stochastic semilinear equations, Stochastic Anal. Appl., 13 (1995), pp. 35-45.

[11] G. Da Prato and J. Zabczyk, Stochastic Equations in Infinite Dimensions, Cambridge University Press, Cambridge, UK, 1992.

[12] G. Da Prato And J. ZabcZYK, Ergodicity for Infinite Dimensional Systems, Cambridge University Press, Cambridge, UK, 1996.

[13] T. E. Duncan, B. Goldys, And B. Pasik-Duncan, Adaptive control of linear stochastic evolution system, Stochastics Stochastics Rep., 36 (1991), pp. 71-90.

[14] T. E. Duncan, B. Maslowski, and B. Pasik-Duncan, Adaptive boundary and point control of linear stochastic distributed parameter systems, SIAM J. Control Optim., 32 (1994), pp. 648-672.

[15] T. E. Duncan, B. Maslowski, and B. Pasik-Duncan, Adaptive boundary control of linear distributed parameter systems described by analytic semigroups, Appl. Math. Optim., 33 (1996), pp. 107-138.

[16] T. E. Duncan, B. Maslowski, And B. Pasik-Duncan, Ergodic boundary/point control of stochastic semilinear systems, SIAM J. Control Optim., 36 (1998), pp. 1020-1047.

[17] T. E. Duncan, B. Pasik-Duncan, and L. Stettner, Almost self-optimizing strategies for the adaptive control of diffusion processes, J. Optim. Theory Appl., 81 (1994), pp. 479-507.

[18] T. E. Duncan, B. Pasik-Duncan, and L. Stettner, On ergodic control of stochastic evolution equations, Stochastic Process. Appl., 15 (1997), pp. 723-750.

[19] K. D. Elworthy, Stochastic Differential Equations on Manifolds, London Math. Soc. Lecture Note Ser. 70, Cambridge University Press, Cambridge, UK 1982.

[20] B. Goldys AND B. MASLOWSKI, Ergodic control of semilinear stochastic equations and Hamilton-Jacobi equations, J. Math. Anal. Appl., 234 (1999), pp. 592-631.

[21] B. Goldys and B. Maslowski, Parameter estimation for controlled semilinear stochastic systems: Identifiability and consistency, J. Multivariate Anal., submitted.

[22] F. Gozzi And E. RouY, Regular solutions to second-order stationary Hamilton-Jacobi equations, J. Differential Equations, 130 (1996), pp. 201-234.

[23] F. Gozzi, E. Rouy, AND A. Swiech, Second-order Hamilton-Jacobi equations in Hilbert spaces and stochastic boundary control, SIAM J. Control Optim., 38 (2000), pp. 400-430.

[24] D. Henry, Geometric Theory of Semilinear Parabolic Equations, Lecture Notes in Math. 840, Springer-Verlag, New York, 1981.

[25] H. J. Kushner, Optimality conditions for the average cost per unit time problem with a diffusion model, SIAM J. Control Optim., 16 (1978), pp. 330-346.

[26] R. S. Liptser and A. N. Shiryayev, Statistics of Random Processes. I: General Theory, Springer-Verlag, New York, 1977. 
[27] B. MASLOWSKI, On probability distributions of solutions of semilinear stochastic evolution equations, Stochastics Stochastics Rep., 45 (1993), pp. 17-44.

[28] M. RoBin, Long-term average cost problems for continuous time Markov processes - a survey, Acta Appl. Math., 1 (1983), pp. 281-300.

[29] J. SeIdler, Ergodic behaviour of stochastic parabolic equations, Czechoslovak Math. J., 47 (1992), pp. 277-316.

[30] J. SeIDlen, Da Prato-Zabczyk maximal inequality revisited I, Math. Bohem., 118 (1993), pp. $67-106$. 